\title{
Coherent-mode decomposition of partially polarized, partially coherent sources
}

\author{
Franco Gori and Massimo Santarsiero \\ Istituto Nazionale per la Fisica della Materia and Dipartimento di Fisica, Università "Roma Tre," Via della \\ Vasca Navale 84, I-00146 Rome, Italy
}

Raja Simon

The Institute of Mathematical Sciences, C.I.T. Campus, Tharamani, Chennai 600 113, India

Gemma Piquero

Departamento de Óptica, Universidad Complutense de Madrid, Ciudad Universitaria s/n, E-28040 Madrid, Spain

Riccardo Borghi and Giorgio Guattari

Istituto Nazionale per la Fisica della Materia and Dipartimento di Elettronica Applicata, Università "Roma Tre," Via della Vasca Navale 84, I-00146 Rome, Italy

Received March 22, 2002; accepted August 26, 2002

\begin{abstract}
It is shown that any partially polarized, partially coherent source can be expressed in terms of a suitable superposition of transverse coherent modes with orthogonal polarization states. Such modes are determined through the solution of a system of two coupled integral equations. An example, for which the modal decomposition is obtained in closed form in terms of fully linearly polarized Hermite Gaussian modes, is given. (C) 2003 Optical Society of America
\end{abstract}

OCIS codes: $260.5430,030.4070$.

\section{INTRODUCTION}

The determination of the coherent $\operatorname{modes}^{1}$ of a partially coherent beam and of their relative weights represents a key problem in characterizing real laser beams and sources, and several methods have been proposed to solve such a problem in the scalar case..$^{2-19}$

In view of the current interest in light beams that are both partially polarized and partially coherent, ${ }^{20-28}$ it is desirable to extend the coherent-mode representation to the case of vectorial electromagnetic beams. In the present paper, we show that such an extension can be carried out in a more or less straightforward way.

The similarity between the coherent-mode representation in the scalar and vectorial cases becomes particularly transparent when the structure of the theory is viewed from a standpoint slightly more formal and general than the position representation, which is more often used in optics. For this reason, we will briefly present this general point of view, showing how the coherent-mode representation of partially coherent scalar sources simply arises as a particular case, and then we will apply the coherent-mode decomposition to the case of partially polarized, partially coherent vectorial beams described by the beam-coherence-polarization (BCP) matrix. ${ }^{25,26}$ Following such a formalism, we will neglect the longitudinal component of the field and consider the paraxial approximation to be valid. We will show that, in general, the determination of the coherent modes of a partially polar- ized, partially coherent source can be a rather difficult task, but it can be made easier if the pertinent BCP matrix can be reduced to a diagonal form. As an example, we will consider partially polarized sources characterized by BCP matrices having Gaussian Schell-model (GSM) ${ }^{1}$ diagonal elements and specular mutual intensity (SMI) ${ }^{29}$ antidiagonal ones.

The paper is organized as follows: In Section 2, the formalism based on the Hilbert space is recalled. After introduction of the notation to be used, such a formalism is applied to the scalar and vectorial cases. In particular, the modal decomposition of a source with a diagonal BCP matrix is derived. In Section 3, such results are applied to the particular case of a source for which closed-form results are achievable. Finally, Section 4 is devoted to the conclusions.

\section{THEORETICAL ANALYSIS}

\section{A. Preliminaries and Notation}

Let $\mathcal{H}$ represent a Hilbert space. Elements of $\mathcal{H}$ are denoted by Dirac's ket vectors $|\varphi\rangle,|\psi\rangle, \ldots$, and the inner product between two vectors $|\varphi\rangle,|\psi\rangle$ is devoted by $\langle\varphi \mid \psi\rangle$. Let $\hat{J}$ be a linear, Hermitian, positive-semidefinite operator acting on $\mathcal{H}$.

Hermiticity means that

$$
\langle\varphi|\hat{J}| \psi\rangle=\langle\psi|\hat{J}| \varphi\rangle^{*}
$$


for all $|\varphi\rangle,|\psi\rangle \in \mathcal{H}$, the asterisk denoting complex conjugation, whereas nonnegativity demands that

$$
\langle\varphi|\hat{J}| \varphi\rangle \geqslant 0
$$

for every $|\varphi\rangle \in \mathcal{H}$. Furthermore, if $\hat{J}$ satisfies the additional condition $\operatorname{Tr}\left\{\hat{J}^{2}\right\}<\infty, \operatorname{Tr}\{\cdot\}$ being the trace operator, then $\hat{J}$ is called a Hilbert-Schmidt operator.

As is well-known, ${ }^{30,31}$ every Hilbert-Schmidt operator has the spectral representation or decomposition

$$
\hat{J}=\sum_{n} \lambda_{n}|n\rangle\langle n|,
$$

where the $\lambda_{n}$ are nonnegative numbers and the kets $|n\rangle$ are orthonormal, i.e.,

$$
\langle m \mid n\rangle=\delta_{m, n},
$$

$\delta_{m, n}$ being the Kronecker symbol. Equation (3) means that $\hat{J}$ is a convex combination of orthogonal onedimensional projection operators in $\mathcal{H}$.

Using the orthonormality property (4), we can rewrite the above representation in the form

$$
\hat{J}|n\rangle=\lambda_{n}|n\rangle, \quad n=0,1,2, \ldots,
$$

so that the $\lambda_{n}$ are the eigenvalues of $\hat{J}$, the corresponding eigenfunctions are (or can be chosen to be) orthonormal, and such eigenfunctions form a basis for the subspace of $\mathcal{H}$ over which $\hat{J}$ is a nonzero operator. In the orthogonal subspace of $\mathcal{H}$, the Hilbert-Schmidt operator acts as a null operator. Clearly, $\operatorname{Tr}\left\{\hat{J}^{2}\right\}=\Sigma_{n} \lambda_{n}{ }^{2}$, and the HilbertSchmidt property gets transcribed into the statement $\Sigma_{n} \lambda_{n}{ }^{2}<\infty$.

The index $n$ runs over a subset of the integer set or the natural numbers. However, in some particular applications, symmetry or other insights may make it natural and convenient to label this subset with two or more discrete variables. Further, a given physical situation may be such that the Hilbert-Schmidt operator of interest is conveniently described in a particular basis, which will be referred to as natural. In that case, the problem will be to determine the eigenvectors of $\hat{J}$ as a linear combination of these preferred, or natural, basis vectors. However, the natural basis may be labeled by one or more discrete variables, and in other cases it is labeled by one or more continuous variables. Indeed, in the vector case, one needs two continuous variables to label the natural basis.

\section{B. Scalar Fields}

In the case of quasi-monochromatic partially coherent light sources described by the mutual intensity $J\left(\mathbf{r}_{1}, \mathbf{r}_{2}\right),{ }^{1,32}$ the natural basis consists of the position eigenvectors $|x, y\rangle=|\mathbf{r}\rangle$, which satisfy the following relationships:

$$
\begin{aligned}
\hat{x}|\mathbf{r}\rangle & =x|\mathbf{r}\rangle, \quad \hat{y}|\mathbf{r}\rangle=y|\mathbf{r}\rangle, \\
\left\langle\mathbf{r}_{1} \mid \mathbf{r}_{2}\right\rangle & =\delta^{(2)}\left(\mathbf{r}_{1}-\mathbf{r}_{2}\right), \\
\int \mathrm{d}^{2} r|\mathbf{r}\rangle\langle\mathbf{r}| & =1 .
\end{aligned}
$$

In Eqs. (6)-(8), $\mathbf{r}_{1}$ and $\mathbf{r}_{2}$ denote two typical points across the transverse section of the beam, while a reference frame $(x, y, z)$, with the $z$ axis coincident with the mean propagation direction of the beam, has been introduced. Furthermore, $\delta^{(2)}$ is the two-dimensional Dirac function.

Given a mutual intensity function $J\left(\mathbf{r}_{1}, \mathbf{r}_{2}\right)$, we represent it as a Hermitian nonnegative operator $\hat{J}$ in the Hilbert space $\mathcal{H}=L_{2}\left(\mathcal{R}^{2}\right)$, which consists of complex-valued functions that are square integrable over the twodimensional plane $\mathcal{R}^{2}$. The matrix elements of $\mathcal{J}$ are defined as

$$
J\left(\mathbf{r}_{1}, \mathbf{r}_{2}\right)=\left\langle\mathbf{r}_{1}|\hat{J}| \mathbf{r}_{2}\right\rangle
$$

Using the completeness property of the position eigenvectors given in Eq. (8), we can immediately invert Eq. (9), i.e.,

$$
\hat{J}=\iint \mathrm{d}^{2} r_{1} \mathrm{~d}^{2} r_{2} J\left(\mathbf{r}_{1}, \mathbf{r}_{2}\right)\left|\mathbf{r}_{1}\right\rangle\left\langle\mathbf{r}_{2}\right| .
$$

Furthermore, it follows that the Hilbert-Schmidt property reads as

$$
\operatorname{Tr}\left\{\hat{J}^{2}\right\}=\iint \mathrm{d}^{2} r_{1} \mathrm{~d}^{2} r_{2}\left|J\left(\mathbf{r}_{1}, \mathbf{r}_{2}\right)\right|^{2}<\infty .
$$

Let us assume that our mutual intensity function possesses such a property. Then the spectral representation theorem applies. On projecting Eq. (3) to the position representation, on using the completeness relation (8), and on denoting $\langle\mathbf{r} \mid n\rangle$, which is a complex-valued scalar function of $\mathbf{r}$, by $\Phi_{n}(\mathbf{r})$, we eventually find that

$$
\begin{gathered}
J\left(\mathbf{r}_{1}, \mathbf{r}_{2}\right)=\sum_{n} \lambda_{n} \Phi_{n}\left(\mathbf{r}_{1}\right) \Phi_{n}^{*}\left(\mathbf{r}_{2}\right), \\
\lambda_{n}>0, \quad(12) \\
\int \mathrm{d}^{2} r \Phi_{m}^{*}(\mathbf{r}) \Phi_{n}(\mathbf{r})=\delta_{m, n} .
\end{gathered}
$$

Similarly, Eq. (5) reads, in the position representation, as follows:

$$
\int \mathrm{d}^{2} r_{2} J\left(\mathbf{r}_{1}, \mathbf{r}_{2}\right) \Phi_{n}\left(\mathbf{r}_{2}\right)=\lambda_{n} \Phi_{n}\left(\mathbf{r}_{1}\right) ;
$$

i.e., it becomes an integral equation.

Equations (12)-(14) indeed coincide with the familiar equations of the coherent-mode representation for partially coherent scalar beams. ${ }^{1,33}$

\section{Vectorial Case}

In the case of vector beams, we need a binary variable, say $\alpha$, to label the states of polarization. Although $|u\rangle$ and $|v\rangle$ may represent two generic orthogonal polarization states, for simplicity we will consider them as linear polarization states along $x$ and $y$, respectively. An arbitrary (fully polarized) state can be written as

$$
c_{u}|u\rangle+c_{v}|v\rangle=\sum_{\alpha} c_{\alpha}|\alpha\rangle,
$$

where $\alpha$ runs over its binary values $u$ and $v$. We wish to allow the possibility that the state of polarization may change with position, so that $c_{u}$ and $c_{v}$ are (independent) functions of position across the transverse plane. It follows that the Hilbert space of relevance to the present 
problem is the tensor product $\mathcal{C}^{2} \otimes L_{2}\left(\mathcal{R}^{2}\right),{ }^{34}$ with $\mathcal{C}^{2}$ representing the two-dimensional complex vector space corresponding to states of polarization and $L_{2}\left(\mathcal{R}^{2}\right)$ again corresponding to the space of complex-valued functions that are square integrable over the plane $\mathcal{R}^{2}$, as in the scalar case. The natural basis is $\{|\alpha ; \mathbf{r}\rangle=|\alpha\rangle \otimes|\mathbf{r}\rangle\}$, where $\alpha$ runs over $(u, v)$ and $\mathbf{r}$ runs over the transverse plane. The orthonormality and completeness relations become

$$
\begin{aligned}
\left\langle\alpha ; \mathbf{r}_{1} \mid \beta ; \mathbf{r}_{2}\right\rangle & =\delta_{\alpha, \beta} \delta^{(2)}\left(\mathbf{r}_{1}-\mathbf{r}_{2}\right), \\
\sum_{\alpha} \int \mathrm{d}^{2} r|\alpha ; \mathbf{r}\rangle\langle\alpha ; \mathbf{r}| & =1,
\end{aligned}
$$

respectively. Furthermore, in Eq. (17), the symbol 1 stands for the identity operator on the Hilbert space $\mathcal{C}^{2}$ $\otimes L_{2}\left(\mathcal{R}^{2}\right)$.

In place of the mutual intensity of the scalar case, we now have the BCP matrix, ${ }^{14,26}$ and thus the BCP operator, denoted again by $\hat{J}$. Then we have the following relationship between the BCP matrix and the BCP operator:

$$
J_{\alpha \beta}\left(\mathbf{r}_{1}, \mathbf{r}_{2}\right)=\left\langle\alpha ; \mathbf{r}_{1}|\hat{J}| \beta ; \mathbf{r}_{2}\right\rangle,
$$

together with the inverse relationship

$$
\hat{J}=\sum_{\alpha, \beta} \mathrm{d}^{2} r_{1} \mathrm{~d}^{2} r_{2} J_{\alpha \beta}\left(\mathbf{r}_{1}, \mathbf{r}_{2}\right)\left|\alpha ; \mathbf{r}_{1}\right\rangle\left\langle\beta ; \mathbf{r}_{2}\right| .
$$

By allowing $\alpha, \beta$ to run over their binary values $(u, v)$ independently, we can write $J_{\alpha \beta}\left(\mathbf{r}_{1}, \mathbf{r}_{2}\right)$ symbolically as a $2 \times 2$ matrix:

$$
\mathbf{J}\left(\mathbf{r}_{1}, \mathbf{r}_{2}\right)=\left[\begin{array}{ll}
J_{u u}\left(\mathbf{r}_{1}, \mathbf{r}_{2}\right) & J_{u v}\left(\mathbf{r}_{1}, \mathbf{r}_{2}\right) \\
J_{v u}\left(\mathbf{r}_{1}, \mathbf{r}_{2}\right) & J_{v v}\left(\mathbf{r}_{1}, \mathbf{r}_{2}\right)
\end{array}\right] .
$$

In particular, the Hilbert-Schmidt property reads now as

$$
\operatorname{Tr}\left\{\hat{J}^{2}\right\}=\sum_{\alpha, \beta} \iint \mathrm{d}^{2} r_{1} \mathrm{~d}^{2} r_{2}\left|J_{\alpha \beta}\left(\mathbf{r}_{1}, \mathbf{r}_{2}\right)\right|^{2}<\infty,
$$

while the nonnegativity condition, which for the $\hat{J}$ operator turns out to be

$$
\langle\varphi|\hat{J}| \varphi\rangle \geqslant 0 \quad \forall|\varphi\rangle \in \mathcal{C}^{2} \otimes L_{2}\left(\mathcal{R}^{2}\right),
$$

leads, once projected to the $|\alpha ; \mathbf{r}\rangle$ basis, to

$$
\sum_{\alpha, \beta} \iint \mathrm{d}^{2} r_{1} \mathrm{~d}^{2} r_{2} J_{\alpha \beta}\left(\mathbf{r}_{1}, \mathbf{r}_{2}\right) \varphi_{\alpha}^{*}\left(\mathbf{r}_{1}\right) \varphi_{\beta}\left(\mathbf{r}_{2}\right) \geqslant 0
$$

for any pair of functions $\varphi_{u}(\mathbf{r}), \varphi_{v}(\mathbf{r})$. We assume henceforth that our BCP matrix (and hence the BCP operator) possesses such a property, so that the spectralrepresentation theorem, given in Eqs. (3)-(5), applies.

Let us denote the function $\langle\alpha ; \mathbf{r} \mid n\rangle$ by $\Phi_{n ; \alpha}(\mathbf{r})$. When $\alpha=u$, this represents a fully coherent beam, fully polarized along the $x$ direction. For $\alpha=v$ the polarization is in the $y$ direction. On projecting Eq. (3) to the $|\alpha ; \mathbf{r}\rangle$ basis and on using the completeness property (17), one obtains

$$
\begin{aligned}
J_{\alpha \beta}\left(\mathbf{r}_{1}, \mathbf{r}_{2}\right) & =\sum_{n} \Lambda_{n} \Phi_{n ; \alpha}\left(\mathbf{r}_{1}\right) \Phi_{n ; \beta}^{*}\left(\mathbf{r}_{2}\right), \\
\sum_{\alpha} \int \mathrm{d}^{2} r \Phi_{n ; \alpha}(\mathbf{r}) \Phi_{n ; \beta}^{*}(\mathbf{r}) & =\delta_{m, n},
\end{aligned}
$$

where $\Lambda_{n}$ denotes the $n$th eigenvalue in the vector case.

The coherent modes now have the vector form

$$
\boldsymbol{\Phi}_{n}(\mathbf{r})=\left(\begin{array}{c}
\Phi_{n ; u}(\mathbf{r}) \\
\Phi_{n ; v}(\mathbf{r})
\end{array}\right)
$$

and $\mathbf{J}$ may be written in the alternative form

$$
\mathbf{J}\left(\mathbf{r}_{1}, \mathbf{r}_{2}\right)=\sum_{n} \Lambda_{n} \Phi_{n}\left(\mathbf{r}_{1}\right) \Phi_{n}^{\dagger}\left(\mathbf{r}_{2}\right),
$$

the dagger denoting the Hermitian conjugate of the vector (26). Furthermore, on projecting Eq. (5) to the $|\alpha ; \mathbf{r}\rangle$ basis, we obtain

$$
\begin{array}{r}
\sum_{\beta} \int \mathrm{d}^{2} r_{2} J_{\alpha \beta}\left(\mathbf{r}_{1}, \mathbf{r}_{2}\right) \Phi_{n ; \beta}\left(\mathbf{r}_{2}\right)=\Lambda_{n} \Phi_{n ; \alpha}\left(\mathbf{r}_{1}\right) \\
(\alpha, \beta=u, v),
\end{array}
$$

which represents a pair of coupled integral equations. This result generalizes the corresponding integral equation (14) (a homogeneous Fredholm integral) obtained for the scalar case. When polarization of light is taken into account, it will be necessary to solve this pair of equations.

For a given $\mathrm{BCP}$ matrix, solving the set of integral equations (28) may be nontrivial. However, in some favorable cases it may happen that a coherent-mode decomposition is known for $J_{u u}, J_{v v}$, and $J_{u v}$ individually and that the integral equations (28) couple only a few of these coherent modes. This happens, for instance, when the BCP matrix has, or can be reduced to, a diagonal form, as we shall see in Subsection 2.D.

\section{Diagonal Case}

Let us suppose that the BCP matrix $\mathbf{J}_{0}$ has a diagonal form, i.e.,

$$
\mathbf{J}_{0}\left(\mathbf{r}_{1}, \mathbf{r}_{2}\right)=\left[\begin{array}{cc}
J_{u u}\left(\mathbf{r}_{1}, \mathbf{r}_{2}\right) & 0 \\
0 & J_{v v}\left(\mathbf{r}_{1}, \mathbf{r}_{2}\right)
\end{array}\right] .
$$

As we shall see in a moment, for such a BCP matrix the modal decomposition can be easily achieved as follows. First, let the modal decompositions of the two diagonal terms of the BCP matrix (29) be

$$
\begin{aligned}
& J_{u u}\left(\mathbf{r}_{1}, \mathbf{r}_{2}\right)=\sum_{n} \lambda_{n} \phi_{n}\left(\mathbf{r}_{1}\right) \phi_{n}^{*}\left(\mathbf{r}_{2}\right), \\
& J_{v v}\left(\mathbf{r}_{1}, \mathbf{r}_{2}\right)=\sum_{n} \mu_{n} \psi_{n}\left(\mathbf{r}_{1}\right) \psi_{n}^{*}\left(\mathbf{r}_{2}\right),
\end{aligned}
$$

respectively. In Eqs. (30) and (31), $\lambda_{n}$ and $\mu_{n}$ are the eigenvalues associated with the two scalar mutual intensities $J_{u u}$ and $J_{v v}$, respectively, while $\phi_{n}(\mathbf{r})$ and $\psi_{n}(\mathbf{r})$ are the corresponding eigenfunctions. Accordingly, the BCP matrix (29) can be given the form in Eq. (27) simply on letting, for instance,

$$
\begin{aligned}
\Lambda_{2 n}=\lambda_{n}, & \boldsymbol{\Phi}_{2 n}(\mathbf{r})=\left(\begin{array}{c}
\phi_{n}(\mathbf{r}) \\
0
\end{array}\right), \\
\Lambda_{2 n+1}=\mu_{n}, & \boldsymbol{\Phi}_{2 n+1}(\mathbf{r})=\left(\begin{array}{c}
0 \\
\psi_{n}(\mathbf{r})
\end{array}\right), \quad n=0,1,2, \ldots,
\end{aligned}
$$


which represents the modal decomposition of a diagonal BCP matrix. In such a way, the modes turn out to be alternately polarized along $x$ and $y$. It should be noted that the diagonal case is of particular importance, since a wide class of BCP matrices can be reduced to a diagonal form simply by means of a suitable rotation of the reference frame. ${ }^{26,27}$

In Section 3, we present an example of modal decomposition of a partially polarized, partially coherent source where the problem can be solved in closed-form terms.

\section{EXAMPLE OF COHERENT-MODE DECOMPOSITION}

\section{A. Preliminaries}

For simplicity, in the following we shall limit ourselves to a two-dimensional problem.

Let us consider the following BCP matrix at the plane $z=0$ of the $(x, z)$ reference frame:

$$
\begin{aligned}
\mathbf{J}_{0}\left(x_{1}, x_{2}\right) & \\
= & I_{0} \exp \left[-\frac{\beta}{2}\left(x_{1}{ }^{2}+x_{2}{ }^{2}\right)\right] \\
& \times\left[\begin{array}{ll}
\exp \left[-\gamma\left(x_{1}-x_{2}\right)^{2}\right] & \exp \left[-\gamma\left(x_{1}+x_{2}\right)^{2}\right] \\
\exp \left[-\gamma\left(x_{1}+x_{2}\right)^{2}\right] & \exp \left[-\gamma\left(x_{1}-x_{2}\right)^{2}\right]
\end{array},\right.
\end{aligned}
$$

where $I_{0}, \beta$, and $\gamma$ are positive parameters. $\quad \mathbf{J}_{0}$ is a bona fide BCP matrix, since it satisfies both the Hermiticity (the matrix is real and symmetric) and nonnegativeness conditions (see Appendix A). On the other hand, the correlation functions between the various components of the field take values having modulus ranging from 0 to 1 .

The partially polarized, partially coherent source characterized by the BCP (33) is indistinguishable, as far as measurements with no anisotropic elements are concerned, from an ordinary, scalar Gaussian Schell-model (GSM) source. ${ }^{1}$ In fact, the mutual intensity of the equivalent scalar partially coherent source ${ }^{35}$ turns out to be

$$
\begin{aligned}
J_{\mathrm{eq}}\left(x_{1}, x_{2}\right)= & 2 I_{0} \exp \left[-\frac{\beta}{2}\left(x_{1}{ }^{2}+x_{2}{ }^{2}\right)\right] \\
& \times \exp \left[-\gamma\left(x_{1}-x_{2}\right)^{2}\right],
\end{aligned}
$$

where both the intensity and the degree of coherence are Gaussian.

Each of the elements of the matrix (33) has the structure of a mutual intensity function. The two diagonal terms simply correspond to the mutual intensity of a scalar GSM source. The antidiagonal ones, on the other hand, present a Gaussian intensity profile, just as for the diagonal elements, but their degree of coherence (which takes into account the cross correlation between $E_{x}$ and $E_{y}$ ) has the form

$$
g\left(x_{1}, x_{2}\right)=\exp \left[-\gamma\left(x_{1}+x_{2}\right)^{2}\right],
$$

i.e., depends only on the sum of the two coordinates $x_{1}$ and $x_{2}$. From Eq. (35), it is easily seen that the maximum value (unitary) of $g$ is achieved for $x_{1}=-x_{2}$, i.e., when the two considered points are symmetric with respect to the origin. Sources of this kind were extensively studied in the scalar case $\mathrm{e}^{29}$ and were termed sources endowed with specular mutual intensity (SMI). In the present case, Eq. (35) leads to the fact that, when $x_{1}$ $=-x_{2}$, a perfect correlation between the components $E_{x}$ and $E_{y}$ is achieved.

As is well-known, the polarization features of the source are determined by the local BCP matrix, i.e., ${ }^{26}$

$$
\mathbf{J}_{0}(x, x)=I_{0} \exp \left(-\beta x^{2}\right)\left[\begin{array}{cc}
1 & \exp \left(-4 \gamma x^{2}\right) \\
\exp \left(-4 \gamma x^{2}\right) & 1
\end{array}\right] .
$$

For instance, let us assume that $|u\rangle$ and $|v\rangle$ correspond to $x$ and $y$ linearly polarized states, respectively, so that the Stokes parameters at each point and the local degree of polarization $P$ (Refs. 1 and 36) are easily calculated from Eq. (36) as

$$
\begin{aligned}
s_{0} & =J_{x x}+J_{y y}=2 I_{0} \exp \left(-\beta x^{2}\right), \\
s_{1} & =J_{x x}-J_{y y}=0, \\
s_{2} & =2 \operatorname{Re}\left(J_{x y}\right)=2 I_{0} \exp \left[-(\beta+4 \gamma) x^{2}\right], \\
s_{3} & =2 \operatorname{Im}\left(J_{x y}\right)=0, \\
P & =\left[\frac{\left(J_{x x}-J_{y y}\right)^{2}+4\left|J_{x y}\right|^{2}}{\left(J_{x x}+J_{y y}\right)^{2}}\right]^{1 / 2}=\exp \left(-4 \gamma x^{2}\right) .
\end{aligned}
$$

The source turns out to be partially polarized with a nonuniform degree of polarization $P$ across the transverse section with a Gaussian profile. In particular, the degree of polarization $P$ presents a Gaussian profile with the maximum value at the center, and the width of such a profile is proportional to $\sqrt{1 / \gamma}$. On the other hand, from the previous equations it can be seen that if we decompose the wave into an unpolarized and a polarized portion that are mutually independent, ${ }^{32}$ then its totally polarized component is linear with azimuth $45^{\circ}$.

An important property of the BCP matrix (33) is that it can be diagonalized simply by using a new reference frame, say $(\xi, \eta)$, for representing the electric field, which is rotated by $\pi / 4$ with respect to the $(x, y)$ fraone. In fact, since in our case $J_{x x}=J_{y y}$ and $J_{x y}=J_{y x}$, the BCP matrix in the $(\xi, \eta)$ reference frame turns out to be $\mathrm{be}^{26}$

$$
\mathbf{J}_{0}\left(x_{1}, x_{2}\right)=\left[\begin{array}{cc}
J_{\xi \xi}\left(x_{1}, x_{2}\right) & 0 \\
0 & J_{\eta \eta}\left(x_{1}, x_{2}\right)
\end{array}\right],
$$

where

$$
\begin{aligned}
J_{\xi \xi}\left(x_{1}, x_{2}\right)= & I_{0} \exp \left[-\frac{\beta}{2}\left(x_{1}^{2}+x_{2}^{2}\right)\right] \\
& \times\left\{\exp \left[-\gamma\left(x_{1}-x_{2}\right)^{2}\right]\right. \\
& \left.+\exp \left[-\gamma\left(x_{1}+x_{2}\right)^{2}\right]\right\}, \\
J_{\eta \eta}\left(x_{1}, x_{2}\right)= & I_{0} \exp \left[-\frac{\beta}{2}\left(x_{1}^{2}+x_{2}^{2}\right)\right] \\
& \times\left\{\exp \left[-\gamma\left(x_{1}-x_{2}\right)^{2}\right]\right. \\
& \left.-\exp \left[-\gamma\left(x_{1}+x_{2}\right)^{2}\right]\right\} .
\end{aligned}
$$


As a consequence, the partially polarized source can be thought of as arising from the superposition of two independent sources, linearly polarized along the $\xi$ and $\eta$ axes, respectively, whose mutual intensities are given by Eqs. (43). Accordingly, results given in Subsection 2D pertinent to diagonal BCP matrices can now be applied to our source. This will be done in the next section.

\section{B. Coherent-Mode Decomposition for Sources with Beam-Coherence-Polarization Matrix of the Form of Eq. (33)}

First, let us introduce the functions $J_{ \pm}\left(x_{1}, x_{2}\right)$, defined as

$$
J_{ \pm}\left(x_{1}, x_{2}\right)=I_{0} \exp \left[-\beta \frac{x_{1}{ }^{2}+x_{2}{ }^{2}}{2}-\gamma\left(x_{1} \pm x_{2}\right)^{2}\right] .
$$

Note that the mutual intensities given in Eqs. (43) can be written in terms of $J_{+}$and $J_{-}$as follows:

$$
\begin{aligned}
& J_{\xi \xi}\left(x_{1}, x_{2}\right)=J_{-}\left(x_{1}, x_{2}\right)+J_{+}\left(x_{1}, x_{2}\right), \\
& J_{\eta \eta}\left(x_{1}, x_{2}\right)=J_{-}\left(x_{1}, x_{2}\right)-J_{+}\left(x_{1}, x_{2}\right) .
\end{aligned}
$$

It is evident that $J_{-}$corresponds to the mutual intensity of a scalar GSM source, so that its coherent-mode decomposition reads ${ }^{37,38}$

$$
\begin{aligned}
J_{-}\left(x_{1}, x_{2}\right)= & \left(\frac{c}{\pi}\right)^{1 / 2} \sum_{n=0}^{\infty} \frac{\lambda_{0} q^{n}}{2^{n} n !} H_{n}\left(x_{1} \sqrt{c}\right) H_{n}\left(x_{2} \sqrt{c}\right) \\
& \times \exp \left(-c \frac{x_{1}^{2}+x_{2}^{2}}{2}\right)
\end{aligned}
$$

where $H_{n}$ is the $n$ th-order Hermite polynomial ${ }^{39}$ and

$$
\begin{aligned}
c & =2\left(\beta^{2}+2 \beta \gamma\right)^{1 / 2}, \\
q & =\gamma /(\beta+\gamma+c), \\
\lambda_{0} & =I_{0}\left(\frac{\pi}{(\beta+\gamma+c)}\right)^{1 / 2} .
\end{aligned}
$$

On the other hand, we can obtain an analogous expansion for $J_{+}$starting from Eq. (46), replacing $x_{2}$ by $-x_{2}$, so that

$$
\begin{aligned}
J_{+}\left(x_{1}, x_{2}\right)= & J_{-}\left(x_{1},-x_{2}\right) \\
= & \left(\frac{c}{\pi}\right)^{1 / 2} \sum_{n=0}^{\infty} \frac{\lambda_{0}(-q)^{n}}{2^{n} n !} H_{n}\left(x_{1} \sqrt{c}\right) \\
& \times H_{n}\left(x_{2} \sqrt{c}\right) \exp \left(-c \frac{x_{1}{ }^{2}+x_{2}{ }^{2}}{2}\right),
\end{aligned}
$$

where use has been made of the following property of the Hermite polynomials ${ }^{39}$ :

$$
H_{n}(-s)=(-1)^{n} H_{n}(s) .
$$

On substituting from Eqs. (46) and (48) into Eq. (45), we finally obtain

$$
\begin{aligned}
J_{\xi \xi}\left(x_{1}, x_{2}\right)= & 2 \lambda_{0}\left(\frac{c}{\pi}\right)^{1 / 2} \sum_{n=0}^{\infty} \frac{q^{2 n}}{2^{2 n} 2 n !} \\
& \times H_{2 n}\left(x_{1} \sqrt{c}\right) H_{2 n}\left(x_{2} \sqrt{c}\right) \\
& \times \exp \left(-c \frac{x_{1}^{2}+x_{2}^{2}}{2}\right), \\
J_{\eta \eta}\left(x_{1}, x_{2}\right)= & 2 \lambda_{0}\left(\frac{c}{\pi}\right)^{1 / 2} \sum_{n=0}^{\infty} \frac{q^{2 n+1}}{2^{2 n+1}(2 n+1) !} \\
& \times H_{2 n+1}\left(x_{1} \sqrt{c}\right) H_{2 n+1}\left(x_{2} \sqrt{c}\right) \\
& \times \exp \left(-c \frac{x_{1}{ }^{2}+x_{2}{ }^{2}}{2}\right),
\end{aligned}
$$

which correspond to the expansions in Eqs. (30) and (31). Accordingly, the modal decomposition (32) holds, on letting

$$
\begin{aligned}
A_{n}= & 2 \lambda_{0} q^{n} \\
\phi_{n}(x)= & \left(\frac{c}{\pi}\right)^{1 / 4} \frac{1}{\sqrt{2^{2 n} 2 n !}} H_{2 n}(x \sqrt{c}) \exp \left(-\frac{c x^{2}}{2}\right), \\
\psi_{n}(x)= & \left(\frac{c}{\pi}\right)^{1 / 4} \frac{1}{\left[2^{2 n+1}(2 n+1) !\right]^{2}} H_{2 n+1}(x \sqrt{c}) \\
& \times \exp \left(-\frac{c x^{2}}{2}\right)
\end{aligned}
$$

which constitutes the coherent-mode decomposition of the partially polarized SMI source. We recall that if the modes of the source are linearly polarized Hermite Gaussian ones, the knowledge of the modal expansion allows us to obtain in an easy way all the beam characzteristics upon propagation through a typical first-order $A B C D$ paraxial optical system. ${ }^{40}$ In particular, since Hermite Gaussian beams are shape invariant upon propagation, the same holds for the beams radiated by sources having a BCP matrix of the form (33). Moreover, in this case the state of polarization, which is described across the source by the Stokes parameters (37)-(40) and by the local transverse degree of polarization, remain unchanged under propagation through first-order optical systems and, in particular, under free propagation. This suggests a method to measure, in a simple way, the coherence features across the source: Simply measure the distribution of polarization [see Eq. (41)] in the transverse section at any plane $z=$ constant.

\section{CONCLUSIONS}

In this paper, a general investigation about the coherentmode decomposition of partially polarized, partially coherent sources has been presented. In doing so, we have applied a formalism based on Hilbert operators to the BCP matrix, which has recently been proposed as a tool for characterizing partially polarized, partially coherent sources. In particular, we have shown that, under very general hypotheses, any partially polarized, partially co- 
herent source can be represented through a superposition of coherent modes with orthogonal polarizations, which have to be determined by solving a system of two coupled integral equations. Such a system decouples in the case of diagonal BCP matrices, so that the problem reduces to two scalar modal decomposition problems.

As a particular case, sources characterized by BCP matrices having GSM diagonal elements and SMI antidiagonal elements have been considered. The beams radiated from these sources are, in general, partially and/or nonuniformly polarized in their transverse section. It turns out that such sources can be expressed as the superposition of fully coherent and linearly polarized Hermite Gaussian modes. As a consequence, the beam keeps the same transverse intensity shape as well as the same local state and degree of polarization under paraxial propagation. Furthermore, it has been shown that the degree of polarization $P$ presents, at any transverse plane, a Gaussian distribution, whose width is related to the coherence features of the source. This fact suggests a method to obtain information about the coherence properties of the source by measuring the sole local degree of polarization at different transverse planes upon free propagation.

\section{APPENDIX A: PROOF OF NONNEGATIVITY}

Let us start from the nonnegativity condition (23), i.e.,

$$
\begin{aligned}
\langle\psi|\hat{J}| \psi\rangle= & \iint \mathrm{d} x_{1} \mathrm{~d} x_{2}\left\{\left[g_{1}^{*}\left(x_{1}\right) g_{1}\left(x_{2}\right)\right.\right. \\
& \left.+g_{2}^{*}\left(x_{1}\right) g_{2}\left(x_{2}\right)\right] \exp \left[-\alpha\left(x_{1}-x_{2}\right)^{2}\right] \\
& +\left[g_{1}^{*}\left(x_{1}\right) g_{2}\left(x_{2}\right)+g_{2}^{*}\left(x_{1}\right) g_{1}\left(x_{2}\right)\right] \\
& \left.\times \exp \left[-\alpha\left(x_{1}+x_{2}\right)^{2}\right]\right\}
\end{aligned}
$$

where we $\operatorname{set} g_{j}(x)=\psi_{j}(x) \exp \left(-\beta x^{2}\right)(j=1,2)$.

If we take into account that

$$
\begin{aligned}
\exp \left[-\alpha\left(x_{1} \pm x_{2}\right)^{2}\right]= & \sqrt{\frac{\pi}{\alpha}} \int \mathrm{d} u \exp \left(-\frac{\pi^{2} u^{2}}{\alpha}\right) \\
& \times \exp \left[i 2 \pi\left(x_{1} \pm x_{2}\right) u\right]
\end{aligned}
$$

Eq. (A1) becomes

$$
\begin{aligned}
\langle\psi|\hat{J}| \psi\rangle= & \sqrt{\frac{\pi}{\alpha}} \int \mathrm{d} u \exp \left(-\frac{\pi^{2} u^{2}}{\alpha}\right) \\
& \times\left[\tilde{g}_{1}^{*}(u) \tilde{g}_{1}(u)+\tilde{g}_{2}^{*}(u) \tilde{g}_{2}(u)\right. \\
& \left.+\tilde{g}_{1}^{*}(u) \tilde{g}_{2}(-u)+\tilde{g}_{2}^{*}(u) \tilde{g}_{1}(-u)\right],
\end{aligned}
$$

where $\tilde{g}_{j}(u)(j=1,2)$ denotes the Fourier transform of the function $g_{j}(x)(j=1,2)$. Let us introduce the even and odd parts of the $\tilde{g}_{j}$ functions, namely,

$$
\widetilde{g}_{j}(u)=\widetilde{E}_{j}(u)+\widetilde{O}_{j}(u), \quad j=1,2,
$$

where, of course, $\widetilde{E}_{j}(-u)=\widetilde{E}_{j}(u)$ and $\tilde{O}_{j}(-u)$ $=-\widetilde{O}_{j}(u)$. On substituting from Eq. (A4) into Eq. (A3), we eventually obtain, after lengthy but straightforward algebra,

$$
\begin{aligned}
\langle\psi|\hat{J}| \psi\rangle= & \sqrt{\frac{\pi}{\alpha}} \int \mathrm{d} u \exp \left(-\frac{\pi^{2} u^{2}}{\alpha}\right)\left[\left|\widetilde{E}_{1}(u)+\widetilde{E}_{2}(u)\right|^{2}\right. \\
& \left.+\left|\widetilde{D}_{1}(u)-\widetilde{D}_{2}(u)\right|^{2}\right],
\end{aligned}
$$

which turns out to be positive for any choice of $f_{j}(x)$.

\section{ACKNOWLEDGMENTS}

One of the authors (G. Piquero) acknowledges support from the project BFM2001-1356.

R. Borghi, the corresponding author, may be reached at riccardo.borghi@uniroma1.it.

\section{REFERENCES}

1. L. Mandel and E. Wolf, Optical Coherence and Quantum Optics (Cambridge U. Press, Cambridge, UK, 1995).

2. R. Gase, T. Gase, and K. Blüthner, "Complex wave-field reconstruction by means of the Page distribution function," Opt. Lett. 20, 2045-2047 (1995).

3. G. Iaconis and I. A. Walmsley, "Direct measurement of the two-point field correlation function," Opt. Lett. 21, 17831785 (1996).

4. J. Tu and S. Tamura, "Analytic relation for recovering the mutual intensity by means of intensity information," J. Opt. Soc. Am. A 15, 202-206 (1998).

5. J. Turunen, E. Tervonen, and A. T. Friberg, "Coherence theoretic algorithm to determine the transverse-mode structure of lasers," Opt. Lett. 14, 627-629 (1989).

6. E. Tervonen, J. Turunen, and A. T. Friberg, "Transverse laser-mode structure determination from spatial coherence measurements: experimental results," Appl. Phys. B 49, 409-414 (1989).

7. A. E. Siegman and S. W. Townsend, "Output beam propagation and beam quality from a multimode stable-cavity laser,” IEEE J. Quantum Electron. 29, 1212-1217 (1993).

8. B. Lü, B. Zhang, B. Cai, and C. Yang, "A simple method for estimating the number of effectively oscillating modes and weighting factors of mixed mode laser beams behaving like Gaussian Schell-model beams," Opt. Commun. 101, 49-52 (1993).

9. A. Cutolo, T. Isernia, I. Izzo, R. Pierri, and L. Zeni, "Transverse mode analysis of a laser beam by near- and far-field intensity measurements," Appl. Opt. 34, 7974-7978 (1995).

10. C. Martínez, F. Encinas-Sanz, J. Serna, P. M. Mejías, and R. Martínez-Herrero, "On the parametric characterization of the transversal spatial structure of laser pulses," Opt. Commun. 139, 299-305 (1997).

11. F. Encinas-Sanz, J. Serna, C. Martínez, R. MartínezHerrero, and P. M. Mejías, "Time-varying beam quality factor and mode evolution in TEA $\mathrm{CO}_{2}$ laser pulses," IEEE J. Quantum Electron. 34, 1835-1838 (1998).

12. C. Martínez, J. Serna, F. Encinas-Sanz, R. MartínezHerrero, and P. M. Mejías, "Time-resolved spatial structure of TEA $\mathrm{CO}_{2}$ laser pulses," Opt. Quantum Electron. 32, 18-30 (2000)

13. R. Borghi and M. Santarsiero, "Modal decomposition of partially coherent flat-topped beams produced by multimode lasers," Opt. Lett. 23, 313-315 (1998).

14. F. Gori, M. Santarsiero, R. Borghi, and G. Guattari, "Intensity-based modal analysis for partially coherent beams with Hermite-Gaussian modes," Opt. Lett. 23, 989991 (1998)

15. M. Santarsiero, F. Gori, R. Borghi, and G. Guattari, "Evaluation of the modal structure for light beams with HermiteGaussian modes," Appl. Opt. 38, 5272-5281 (1999). 
16. R. Borghi and M. Santarsiero, "Modal structure analysis for a class of axially symmetric flat-topped laser beams," IEEE J. Quantum Electron. 35, 745-750 (1999)

17. M. Santarsiero, F. Gori, and R. Borghi, "Modal-weight determination for a class of multimode beams," in Laser Beam and Optics Characterization, H. Weber and H. Laabs, eds. (Optisches Institut, Technische Universität Berlin, Berlin, 2000), pp. 161-170.

18. X. Xue, H. Wei, and A. G. Kirk, "Intensity-based modal decomposition of optical beams in terms of Hermite-Gaussian functions," J. Opt. Soc. Am. A 17, 1086-1091 (2000).

19. H. Laabs, B. Eppich, and H. Weber, "Modal decomposition of partially coherent beams using the ambiguity function," J. Opt. Soc. Am. A 19, 497-504 (2002).

20. D. F. V. James, "Change of polarization of light beam on propagation in free space," J. Opt. Soc. Am. A 11, 1641-1643 (1994).

21. D. F. V. James, "Polarization of light radiated by black-body sources," Opt. Commun. 109, 209-214 (1994).

22. C. Olson, P. L. Greene, G. W. Wicks, D. G. Hall, and S. Rishton, "High-order azimuthal spatial modes of concentriccircle-grating surface-emitting semiconductor lasers," Appl. Phys. Lett. 72, 1284-1286 (1998).

23. R. Martínez-Herrero, P. M. Mejías, and J. M. Movilla, "Spatial characterization of partially polarized beams," Opt. Lett. 22, 206-208 (1997).

24. A. A. Tovar, "Production and propagation of cylindrically polarized Laguerre Gaussian laser beams," J. Opt. Soc. Am. A 15, 2705-2711 (1998)

25. F. Gori, "Matrix treatment for partially polarized, partially coherent beams," Opt. Lett. 23, 241 (1998).

26. F. Gori, M. Santarsiero, S. Vicalvi, R. Borghi, and G. Guattari, "Beam coherence-polarization matrix," J. Eur. Opt. Soc. A Pure Appl. Opt., 7, 941-951 (1998).
27. F. Gori, M. Santarsiero, G. Piquero, R. Borghi, A. Mondello, and R. Simon, "Partially polarized Gaussian Schell-model beams," J. Opt. Pure Appl. Opt. 3, 1-9 (2001).

28. J. $\mathrm{Pu}$ and $\mathrm{B}$. Lu, "Focal shifts in focused nonuniformly polarized beams," J. Opt. Soc. Am. A 18, 2760-2766 (2001)

29. F. Gori, G. Guattari, C. Palma, and C. Padovani, "Specular cross-spectral density functions," Opt. Commun. 68, 239243 (1988).

30. S. K. Berberian, Introduction to Hilbert Space (Oxford U. Press, Oxford, UK, 1961).

31. E. Kreyszig, Introductory Functional Analysis with Applications (Wiley, New York, 1978).

32. M. Born and E. Wolf, Principles of Optics, 7th ed. (Cambridge U. Press, Cambridge, UK, 1999).

33. E. Wolf, "New theory of partial coherence in the spacefrequency domain. Part I: Spectra and cross-spectra of steady-state sources," J. Opt. Soc. Am. 72, 343-351 (1982).

34. C. Cohen-Tannoudji, B. Diu, and F. Laloe, Quantum Me chanics (Wiley, Paris, 1977).

35. F. Gori, M. Santarsiero, R. Borghi, and G. Guattari, "The irradiance of partially polarized beams in a scalar treatment," Opt. Commun. 163, 159-163 (1999).

36. C. Brosseau, Fundamentals of Polarized Light (Wiley, New York, 1998).

37. F. Gori, "Collett-Wolf sources and multimode laser," Opt. Commun. 34, 301-305 (1980)

38. A. Starikov and E. Wolf, "Coherent-mode representation of Gaussian Schell-model sources and of their radiation fields," J. Opt. Soc. Am. 72, 923-928 (1982).

39. M. Abramowitz and I. Stegun, Handbook of Mathematical Functions (Dover, New York, 1972).

40. F. Gori, "Mode propagation of the field generated by ColletWolf Schell-model sources," Opt. Commun. 46, 149-154 (1983). 